\title{
Utility of lavage cytology plus targeted biopsy during cholangioscopy for the diagnosis of indeterminate biliary lesions
}

\author{
Yasuaki Motomura $^{1 *}$, Kazuya Akahoshi ${ }^{1}$, Kiyoshi Kajiyama ${ }^{2}$, Junya Gibo $^{1}$, Kazuaki Miyamoto ${ }^{1}$, Hiroko Ikeda ${ }^{1}$, Eriko Yamaguchi ${ }^{1}$, Katsuhito \\ Teramatsu $^{1}$, Ran Utsunomiya ${ }^{1}$, Aki Miyagaki ${ }^{1}$, Masafumi Ooya ${ }^{3}$ and Eikichi Ihara ${ }^{4}$ \\ ${ }^{1}$ Department of Gastroenterology, Aso Iizuka Hospital, Iizuka, Japan \\ ${ }^{2}$ Department of Surgery, Aso Iizuka Hospital, Iizuka, Japan \\ ${ }^{3}$ Department of Pathology, Aso Iizuka Hospital, Iizuka, Japan \\ ${ }^{4}$ Department of Medicine and Bioregulatory Science, Graduate School of Medical Sciences, Kyushu University, Fukuoka, Japan
}

\begin{abstract}
Background: Cholangioscopy-guided targeted tissue biopsy is reportedly more accurate than conventional brush cytology for the diagnosis of indeterminate biliary lesions. However, the diagnostic value of targeted biopsy alone is yet to be satisfiable.

Aims: In the present study, we determined the utility of cytological analysis of lavage samples collected from the biliary tract during cholangioscopy in combination with targeted biopsy for the diagnosis of biliary lesions.

Methods: Medical records of 59 patients with indeterminate biliary lesions who underwent single-operator cholangioscopy between July 2011 and January 2016 were reviewed. During cholangioscopy, lavage fluid was collected for cytological examination, followed by targeted biopsy of the lesions. Forty out of 59 patients who underwent both biliary lavage cytology and targeted biopsy were retrospectively analyzed. Cytological and/or histological findings were compared with the final diagnosis that was reached by surgery or by clinical follow-up over a period of at least 12 months in non-surgical patients.
\end{abstract}

Results: Diagnostic yields of targeted biopsy, biliary lavage cytology, and targeted biopsy in combination with biliary lavage cytology were as follows: sensitivity, 69.7\%/69.7\%/87.9\%; specificity, 100\%/100\%/100\%; positive predictive value, 100\%/100\%/100\%; negative predictive value, $41.2 \% / 41.2 \% / 63.6 \%$; and diagnostic accuracy, $75.0 \% / 75.0 \% / 90.0 \%$, respectively.

Conclusions: Biliary lavage cytology using single-operator cholangioscopy was easy-to-perform and safe. Our findings suggested that biliary lavage cytology in combination with targeted biopsy using single-operator cholangioscopy might improve the overall diagnostic yield of biliary lesions.

\section{Introduction}

The diagnosis of indeterminate biliary lesions remains challenging [1]. Although widely performed, the sensitivity of biliary brush cytology is not sufficiently high, and its accuracy is only $43.0-66.7 \%$ $[2,3]$. The most recent meta-analysis reported that brush cytology for malignant biliary strictures had a sensitivity of $45.0 \%$, specificity of $99.0 \%$, and diagnostic odds ratio (DOR) of 33.43 [3]. Low cellular yields are considered as the primary reason for the low sensitivity of brush cytology. Hence, various techniques have been employed to improve the sensitivity of biliary lesion diagnosis, including fluorescence in situ hybridization [4], increasing cellular yield via application of a new brush type [5,6], a new cell block technique [7], and a pre-dilation technique to disrupt strictures [8].

Outcomes of transpapillary biopsy, which was utilized to address the low sensitivity of biliary lesion diagnosis and to collect histological evidence, were not as good as anticipated [9]. A recent meta-analysis determined that transpapillary biopsy for malignant biliary strictures had a sensitivity of $48.1 \%$, specificity of $99.2 \%$, and DOR of 43.18 , which were comparable to those of brush cytology [3]. However, combination of brush cytology with transpapillary biopsy increased the sensitivity to $59.4 \%$ and the specificity to $100 \%[3]$.
Peroral cholangioscopy is a useful modality for the diagnosis of biliary lesions. The SpyGlass ${ }^{\circledR}$ system by Boston Scientific is a recently launched single-operator cholangioscopy (SOCS) [10]. The sensitivity, specificity, and accuracy of targeted biopsy (TB) of biliary strictures using SpyGlass ${ }^{\circledR}$ were reported as $49-88 \%, 82-100 \%$, and $25-88 \%$, respectively [11-19]. However, a meta-analysis showed that the sensitivity of the SpyGlass ${ }^{\circledR}$ system was only moderate; thus, its diagnostic value requires improvement [20]. One potential approach for improving the diagnostic value of SpyBite ${ }^{\circledR} \mathrm{TB}$, is improving the technique and/or device for greater cellular yield. Another approach is the combined use of another modality such as cytological examination. Varadarajulu et al. [21] recently reported that rapid onsite evaluation

Correspondence to: Yasuaki Motomura, Department of Gastroenterology, Tokyo Bay Urayasu Ichikawa Medical Center, 3-4-32 Todaijima, Urayasu 2790001, Japan, Tel: +81-47-351-3101; Fax: +81-47-352-6237; E-mail: yasumm@ gmail.com

Key words: biopsy; cholangiocarcinoma; cholangioscopy; cytology; lavage

Received: October 04, 2017; Accepted: October 17, 2017; Published: October 23,2017 
of touch imprint cytology improved the diagnostic outcomes of SOCSguided biopsy in indeterminate biliary strictures. In a previous study, we utilized cytological assessment of lavage fluid during SpyGlass ${ }^{\circledR}$ examination (unpublished study). In this study, we investigated the efficacy of cytological analysis of lavage samples collected from the biliary tract during cholangioscopy, in combination with targeted biopsy, for the diagnosis of biliary lesions. Herein, we report the utility of SpyBite ${ }^{\circledR}$ TB in combination with biliary lavage cytology (BLC) for the diagnosis of indeterminate biliary lesions.

\section{Methods}

\section{Patients}

We retrospectively reviewed the medical records, endoscopic, radiologic and pathologic findings, and clinical outcomes of 59 patients with indeterminate biliary lesions who underwent SOCS between July 2011 and January 2016. After excluding 16 patients who underwent only TB or BLC, the final cohort included 40 patients who underwent both TB and BLC. Hyperbililubinemia was seen in 10, and cholangitis was seen in six out of 40 patients. The study protocol was approved by the institutional review board of Aso Iizuka Hospital in Iizuka, Japan. Written informed consent for endoscopic retrograde cholangiopancreatography (ERCP), including collection of tissue samples via TB and BLC during SOCS, was obtained from all patients. Cytological and/or histological findings of each ERCP specimen were compared with the final diagnosis, which was reached during surgery or clinical follow-up of at least 12 months in non-surgical patients. In some patients, standard brush cytology (BC; RX cytology brush: Boston Scientific Japan) was not performed in the same session; however, it was previously implemented in some patients; $\mathrm{BC}$ results were also compared among those patients.

\section{Endoscopic retrograde cholangio-pancreatography and peroral cholangioscopy}

All ERCP procedures were performed with digital fluoroscopic assistance in patients under conscious sedation by intravenous administration of $35 \mathrm{mg}$ pethidine hydrochloride and $0.5-2 \mathrm{mg}$ flunitrazepam. A C-vision Safire ${ }^{\circledR}$ or Sonialvision Safire ${ }^{\circledR}$ digital fluoroscope (Shimadzu Corporation, Kyoto, Japan) and a JF-260 or TJF-240 video endoscope (Olympus, Tokyo, Japan) with $\mathrm{CO}_{2}$ instead of standard air insufflation were used in all procedures [22].

Peroral cholangioscopy was performed using the SpyGlass ${ }^{\circledR}$ Direct Visualization System (Boston Scientific Japan, Tokyo, Japan). Deep cannulation of the bile duct followed by cholangiography was performed to identify the biliary stricture (Figure 1A). A guidewire (Jagwire, 0.035 inch; Boston Scientific Japan) was replaced, and endoscopic sphincterotomy was performed. The SpyGlass ${ }^{\circledR}$ was then inserted over a guidewire to visualize the stricture (Figure 1B). During cholangioscopy, saline was injected from the irrigation port of the SpyScope ${ }^{\circledR}$ using an adjunct electrical pump, and lavage fluid was removed from the device port of the SpyScope ${ }^{\circledR}$ by suction using a syringe by hand or with the use of a standard electrical pump. Approximately 100-200 ml lavage fluid was collected in one session. The volume of lavage fluid collected was dependent on the time scale of the procedure. The collected lavage fluid was sent to the cytology lab immediately after the procedure and processed according to standard fluid cytology protocols (Figure 1C). Each specimen was evaluated by a pathologist and assigned one of the following six classifications: malignancy/positive, suspected malignancy, neoplastic, atypical, benign/negative, or non-diagnostic/inadequate [23]. Malignancy and
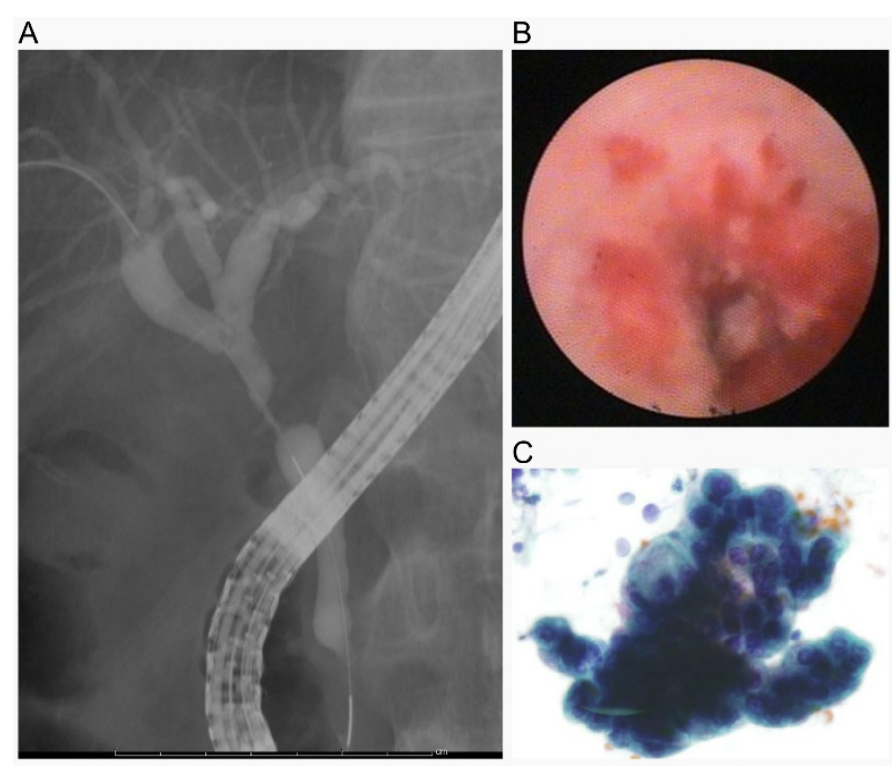

Figure 1. (A) Cholangiography showing a severe stricture in the perihilar bile duct and dilation of the hepatic ducts. (B) SpyGlass ${ }^{\circledR}$ is inserted into the common bile duct to visualize the duodenal side of the stricture. (C) Atypical cells are observed in the collected biliary lavage obtained via peroral cholangioscopy.

suspected malignancy were considered positive in this study. TB of the stricture was performed using SpyBite ${ }^{\circledR}$ forceps until two visible tissue samples were obtained. Antibiotics were not administered prior to cholangioscopy.

\section{Statistical analysis}

The diagnostic accuracy of TB, BLC, TB in combination with BLC, and $\mathrm{BC}$ alone, as well as corresponding probability $(p)$ values, were calculated using the SAS software v94 (SAS Institute, Cary, NC, USA). DOR was calculated as an approximate value, with a false positive value of 0.5. The McNemar test was performed using EZR software to compare the diagnostic values of TB, BLC, TB in combination with $\mathrm{BLC}$, and $\mathrm{BC}$ alone. A $p$ value of $\leq 0.05$ was considered statistically significant for all analyzes performed.

\section{Results}

Average age in this study cohort of 40 patients $(20$ men and 20 women) was $72.6 \pm 10.3$ years. The final diagnoses were intrahepatic cholangiocarcinoma, perihilar cholangiocarcinoma, extrahepatic cholangiocarcinoma, gall bladder cancer, benign stricture/cholangitis, intraductal papillary neoplasm of the bile duct, cystic duct neoplasm, malignant lymphoma, and hepatocellular carcinoma in 5, 9, 13, 2, 7, $1,1,1$, and 1 patients, respectively. The final pathological diagnoses in 21 patients $(52.5 \%)$ who underwent surgery were intrahepatic cholangiocarcinoma, perihilar cholangiocarcinoma, extrahepatic cholangiocarcinoma, gall bladder cancer, intraductal papillary neoplasm of the bile duct, cystic duct neoplasm, and hepatocellular carcinoma in 4, 3, 9, 2, 1, 1, and 1 patients, respectively.

\section{Diagnostic yield of TB, BLC, and TB in combination with BLC}

Biliary lesions were visualized by SpyGlass ${ }^{\circledR}$ in all cases. SpyScope ${ }^{\circledR}$ passed the stricture in 28 out of the 40 cases. SpyGlass ${ }^{\circledR}$ failed to pass the stricture in $5,4,1,1$, and 1 patients with intrahepatic cholangiocarcinoma, perihilar cholangiocarcinoma, intraductal papillary neoplasm of the bile duct, malignant lymphoma, and 
hepatocellular carcinoma, respectively. Adequate samples for both histological and cytological evaluations were obtained in all cases. The diagnostic yields of TB, BLC, and TB in combination with BLC were as follows: sensitivity, $69.7 \% / 69.7 \% / 87.9 \%$; specificity, $100 \% / 100 \% / 100 \%$; diagnostic accuracy, 75.0\%/75.0\%/90.0\%; positive predictive value, $100 \% / 100 \% / 100 \%$; negative predictive value, $41.2 \% / 41.2 \% / 63.6 \%$; and DOR, 32.2\%/32.2\%/101.5\%, respectively (Table 1). Albeit not significant, there was a trend between TB and TB in combination with BLC $(p=0.07)$. The diagnostic yield of $\mathrm{BC}$, which was performed in 17 out of the 40 cases, was as follows: sensitivity, $53.8 \%$; specificity, $75.0 \%$; diagnostic accuracy, $58.8 \%$; positive predictive value, $87.5 \%$; negative predictive value, $33.3 \%$; and DOR, $3.5 \%$.

\section{Tumor localization and diagnostic accuracy}

$\mathrm{TB}$ and BLC positivity rates in cholangiocarcinoma cases were analyzed. TB was positive in 40\% (2/5), 89\% (8/9), and 85\% (11/13) of the intrahepatic, perihilar, and extrahepatic cholangiocarcinoma cases, respectively. In contrast, BLC was positive in $60 \%$ (3/5), $56 \%$ $(5 / 9)$, and $85 \%(11 / 13)$ of the intrahepatic, perihilar, and extrahepatic cholangiocarcinoma cases, respectively (Table 2 ).

Six cases were TB-negative and BLC-positive, which included $1,1,1$, and 3 cases of intrahepatic cholangiocarcinoma, perihilar cholangiocarcinoma, extrahepatic cholangiocarcinoma, and other diagnoses, respectively. Conversely, there were four patients with perihilar cholangiocarcinoma, one patient with extrahepatic cholangiocarcinoma, and one patient with another diagnosis that were TB-positive and BLC-negative. Four cases that were negative by both TB and BLC included two patients with intrahepatic cholangiocarcinoma, one patient with extrahepatic cholangiocarcinoma, and one patient with another diagnosis (Table 3).

Table 1. Comparison of the diagnostic accuracy of targeted biopsy (TB), biliary lavage cytology (BLC), and TB in combination with BLC. ${ }^{*}$ : Diagnostic odds ratio = true positive $(\mathrm{TP}) /$ false negative $(\mathrm{FN}) \div$ false positive $(\mathrm{FP}) /$ true negative $(\mathrm{TN})$. Approximate value was calculated as a FP value of 0.5 . P value was calculated using the SAS ver. 9.4

\begin{tabular}{|c|c|c|c|}
\hline & TB & BLC & TB + BLC \\
\hline Sensitivity (\%) & 69.7 & 69.7 & 87.9 \\
\hline Specificity (\%) & 100 & 100 & 100 \\
\hline Accuracy (\%) & 75.0 & 75.0 & 90.0 \\
\hline Positive predictive value (\%) & 100 & 100 & 100 \\
\hline Negative predictive value (\%) & 41.2 & 41.2 & 63.6 \\
\hline Likelihood ratio + & - & - & - \\
\hline Likelihood ratio - & 0.30 & 0.30 & 0.12 \\
\hline Diagnostic odds ratio* & 32.2 & 32.2 & 101.5 \\
\hline$P$ & $<0.001$ & $<0.001$ & $<0.001$ \\
\hline
\end{tabular}

Table 2. Diagnostic ability of targeted biopsy (TB) and biliary lavage cytology (BLC) in cholangiocarcinoma at different regions.

\begin{tabular}{|c|c|c|c|}
\hline & Intrahepatic & Perihilar & Extrahepatic \\
\hline TB-positive & $40 \%(2 / 5)$ & $89 \%(8 / 9)$ & $85 \%(11 / 13)$ \\
\hline BLC-positive & $60 \%(3 / 5)$ & $56 \%(5 / 9)$ & $85 \%(11 / 13)$ \\
\hline
\end{tabular}

Table 3. Test negative cases by targeted biopsy (TB) and/or biliary lavage cytology (BLC). CC: cholangiocarcinoma; *: one intraductal papillary neoplasm of the bile duct, one cystic duct neoplasm, and one hepatocellular carcinoma; **: malignant lymphoma; ***: gall bladder cancer.

\begin{tabular}{|l|c|c|c|c|}
\hline & $\begin{array}{c}\text { Intrahepatic } \\
\text { CC }\end{array}$ & Perihilar CC & $\begin{array}{c}\text { Extrahepatic } \\
\text { CC }\end{array}$ & Others \\
\hline TB-/BLC+ $(\mathrm{n}=6)$ & 1 & 1 & 1 & $3 *$ \\
\hline TB+/BLC- $(\mathrm{n}=6)$ & 0 & 4 & 1 & $1 * *$ \\
\hline TB-/BLC- $(\mathrm{n}=4)$ & 2 & 0 & 1 & $1 * * *$ \\
\hline
\end{tabular}

\section{Adverse events}

Procedure-related adverse events occurred in 7 (17.5\%) out of the 40 patients and included mild cholangitis in three (7.5\%) patients and mild pancreatitis in four (10\%) patients. Pancreatitis occurred in one patient with intrahepatic cholangiocarcinoma, two patients with perihilar cholangiocarcinoma, and one patient with benign stricture. Cholangitis occurred in two patients with perihilar cholangiocarcinoma and one patient with extrahepatic cholangiocarcinoma. All events resolved with conservative treatment.

\section{Discussion and conclusion}

The present study is the first to demonstrate the efficacy of BLC during SOCS. The diagnostic yield of BLC was comparable to that of TB. BLC under choledochoscopy was reported by Nishimura et al. [24]. However, BLC is not widely used probably because percutaneous choledochoscopy and/or transpapillary cholangioscopy are not popular techniques and biliary lavage is relatively challenging. The SpyGlass ${ }^{\circledR}$ system is equipped with an independent irrigation channel to aspire fluid via a working channel, although it does not have a default suction system [10]. In this cohort, aspiration was achieved by hand or with the use of an electric pump during SOCS to prevent post-procedure cholangitis. Irrigation during cholangioscopy is suggested to increase intrabiliary pressure and promote cholangitis [25]. All three cases of cholangitis in this study were mild. We predict that the effect of suction during the procedure may have prevented severe cholangitis. Aspiration during SOCS may also aid in improving visualization and reducing procedure time.

The diagnostic accuracy of BLC was better than that of BC [3]. One reason for this finding might be the distortion of the stricture by the SpyGlass ${ }^{\circledR}$ system. Balloon predilation was previously reported to significantly improve the diagnostic yield of BC [8]. Sugimoto et al. [26] recently reported that the diagnostic efficacy of cytological evaluation of post-brushing biliary lavage fluid was superior to that of BC in bile duct cancer ( $75 \%$ vs. $49 \%)$. We predict that the effect of lavage followed by dilation/distortion by SpyGlass ${ }^{\circledR}$ might be comparable to that achieved by predilation or post-brushing lavage.

The diagnostic yield of TB under SOCS in the current study was comparable to that reported by previous studies [11-19]. Many of the previous reports noted that more than three tissue samples were required for an accurate diagnosis, as the relatively small tissue samples obtained by SpyBite ${ }^{\circledR}$ rendered pathological examination challenging. In the present study, at least two visible tissue samples were obtained using $\mathrm{TB}$ to reduce the procedure time. It was often necessary to perform TB to collect specimens not only from the primary lesion but also from multiple different sites for mapping biopsies. As TB of several sites will be time consuming in cases where more than three biopsies per site are required, BLC might also compensate for the reduced number of TBs.

Conversely, the diagnostic power appears to differ among lesion locations. The accuracy of TB, which was high for perihilar and distal lesions, was low for intrahepatic lesions, probably because an accurate biopsy might be difficult to achieve owing to the curvature of the bile duct. BLC had a higher accuracy for distal lesions compared with intrahepatic and perihilar lesions, which might be related to the occasionally encountered difficulty in passing the SpyGlass ${ }^{\circledR}$ through a perihilar or intrahepatic lesion. Therefore, there was no distortion effect. Thus, we believe that TB in combination with BLC might be useful for intrahepatic and perihilar cholangiocarcinoma cases, which are often difficult to diagnose with only TB or BLC. In this cohort, 
four cases were TB- and BLC-negative and included two intrahepatic cholangiocarcinoma and one extrahepatic cholangiocarcinoma cases. Improvement of diagnostic devices and/or modalities are sorely required for such cases.

In the present study, there was no case of pancreatic cancer, and cholangioscopy was not performed for suspicious pancreatic cancer; these cases were examined with other modalities such as computed tomography or magnetic resonance imaging. As suspicious lesions were usually observed as mass lesions of the pancreatic head, pancreatic cancer cases were often diagnosed by endoscopic ultrasound-guided fine needle aspiration at our institute. Diagnosis of pancreatic cancer with the BLC technique might be challenging as it usually does not expose the surface of the bile duct.

The recently launched SpyGlassDS ${ }^{\circledast}$ direct visualization system, a digital SOCS, was reported to be useful for diagnosis with both high-quality images and TB $[27,28]$. One of the innovations of this new system is the ability to perform aspiration. Besides irrigation, aspiration is also possible using a Y-port adapter [27]. This system can also improve visualization and reduce the procedure time. An additional electrical pump is required for effective aspiration with the former SpyGlass ${ }^{\circledR}$ system but not with SpyGlassDS ${ }^{\circledR}$, which also allows for easy implementation of BLC. The diagnostic yield of SpyGlassDS ${ }^{\circledR}$ for indeterminate biliary disorders was high, although achieving $100 \%$ accuracy might be difficult. Therefore, BLC could be a useful diagnostic approach even with the new SpyGlassDS ${ }^{\circledR}$ system.

In conclusion, BLC during SOCS was an easy-to-perform, safe, and useful approach to support the diagnosis of indeterminate biliary lesions by TB. As SOCS may be widely performed in high-volume centers and other general hospitals in the near future, BLC may become a necessary technique. However, the present study was was a retrospective study performed in a single institute with a limited number of cases. Therefore, further investigations, such as prospective controlled studies, are required.

\section{References}

1. Victor DW, Sherman S, Karakan T, Khashab MA (2012) Current endoscopic approach to indeterminate biliary strictures. World J Gastroenterol 18: 6197-205. [Crossref]

2. Volmar KE, Vollmer RT, Routbort MJ, Creager AJ (2006) Pancreatic and bile duct brushing cytology in 1000 cases: review of findings and comparison of preparation methods. Cancer 108: 231-238. [Crossref]

3. Navaneethan U, Njei B, Lourdusamy V, Konjeti R, Vargo JJ, et al. (2015) Comparative effectiveness of biliary brush cytology and intraductal biopsy for detection of malignant biliary strictures: a systematic review and meta-analysis. Gastrointest Endosc 81: 168176. [Crossref]

4. Smoczynski M, Jablonska A, Matyskiel A, Lakomy J, Dubowik M, et al (2012) Routine brush cytology and fluorescence in situ hybridization for assessment of pancreatobiliary strictures. Gastrointest Endosc 75: 65-73. [Crossref]

5. Fogel EL, deBellis M, McHenry L, Watkins JL, Chappo J, et al. (2006) Effectiveness of a new long cytology brush in the evaluation of malignant biliary obstruction: a prospective study. Gastrointest Endosc 63: 71-77. [Crossref]

6. Shieh FK, Luong-Player A, Khara HS, Liu H, Lin F, et al. (2014) Improved endoscopic retrograde cholangiopancreatography brush increases diagnostic yield of malignant biliary strictures. World J Gastrointest Endosc 6: 312-317. [Crossref]

7. Noda Y, Fujita N, Kobayashi G, Ito K, Horaguchi J, et al. (2013) Prospective randomized controlled study comparing cell block method and conventional smear method for bile cytology. Dig Endosc 25: 444-452. [Crossref]

8. Farrell RJ, Jain AK, Brandwein SL, Wang H, Chuttani R, et al. (2001) The combination of stricture dilation, endoscopic needle aspiration, and biliary brushings significantly improves diagnostic yield from malignant bile duct strictures. Gastrointest Endosc 54: 587-594. [Crossref]

9. Heinzow HS, Woestmeyer C, Domschke W, Domagk D, Meister T (2013) Endoscopic transpapillary biopsies are of limited value in the diagnostics of bile duct strictures of unknown etiology-results of a histopathologically controlled study in 312 patients. Hepato-gastroenterology. 60: 1569-1572. [Crossref]
10. Chen YK, Pleskow DK (2007) SpyGlass single-operator peroral cholangiopancreatoscopy system for the diagnosis and therapy of bile-duct disorders: a clinical feasibility study (with video). Gastrointest Endosc 65: 832-841. [Crossref]

11. Draganov PV, Lin T, Chauhan S, Wagh MS, Hou W, et al. (2011) Prospective evaluation of the clinical utility of ERCP-guided cholangiopancreatoscopy with a new direct visualization system. Gastrointest Endosc 73: 971-979. [Crossref]

12. Ramchandani M, Reddy DN, Gupta R, Lakhtakia S, Tandan M, et al. (2011) Role of single-operator peroral cholangioscopy in the diagnosis of indeterminate biliary lesions: a single-center, prospective study. Gastrointest Endosc 74: 511-519. [Crossref]

13. Siddiqui AA, Mehendiratta V, Jackson W, Loren DE, Kowalski TE, et al. (2012) Identification of cholangiocarcinoma by using the Spyglass Spyscope system for peroral cholangioscopy and biopsy collection. Clin Gastroenterol Hepatol 10: 466471. [Crossref]

14. Kalaitzakis E, Webster GJ, Oppong KW, Kallis Y, Vlavianos P, et al. (2012) Diagnostic and therapeutic utility of single-operator peroral cholangioscopy for indeterminate biliary lesions and bile duct stones. Eur J Gastroenterol Hepatol 24: 656-664. [Crossref]

15. Manta R, Frazzoni M, Conigliaro R, Maccio L., Melotti G, et al. (2013) SpyGlass singleoperator peroral cholangioscopy in the evaluation of indeterminate biliary lesions: a single-center, prospective, cohort study. Surg Endosc 27: 1569-1572. [Crossref]

16. Rey JW, Hansen T, Dumcke S, Tresch A, Kramer K, et al. (2014) Efficacy of SpyGlass(TM)-directed biopsy compared to brush cytology in obtaining adequate tissue for diagnosis in patients with biliary strictures. World J Gastrointest Endosc 6: 137-143. [Crossref]

17. Woo YS, Lee JK, Oh SH, Kim MJ, Jung JG, et al. (2014) Role of SpyGlass perora cholangioscopy in the evaluation of indeterminate biliary lesions. Dig Dis Sci 59: 25652570. [Crossref]

18. Tieu AH, Kumbhari V, Jakhete N, Onyimba F, Patel Y, et al. (2015) Diagnostic and therapeutic utility of SpyGlass((R)) peroral cholangioscopy in intraductal biliary disease: single-center, retrospective, cohort study. Dig Endosc 27: 479-485. [Crossref]

19. Kurihara T, Yasuda I, Isayama H, Tsuyuguchi T, Yamaguchi T, et al. (2016) Diagnostic and therapeutic single-operator cholangiopancreatoscopy in biliopancreatic diseases: Prospective multicenter study in Japan. World J of Gastroenterol 22: 1891-1901. [Crossref]

20. Navaneethan U, Hasan MK, Lourdusamy V, Njei B, Varadarajulu S, et al. (2015) Single-operator cholangioscopy and targeted biopsies in the diagnosis of indeterminate biliary strictures: a systematic review. Gastrointest Endosc 82: 608-614. [Crossref]

21. Varadarajulu S, Bang JY, Hasan MK, Navaneethan U, Hawes R, et al. (2016) Improving the diagnostic yield of single-operator cholangioscopy-guided biopsy of indeterminate biliary strictures: ROSE to the rescue? (with video). Gastrointest Endosc 84: 681-687. [Crossref]

22. Motomura Y, Akahoshi K, Gibo J, Kanayama K, Fukuda S, et al. (2014) Immediate detection of endoscopic retrograde cholangiopancreatography-related periampullary perforation: fluoroscopy or endoscopy? World J Gastroenterol 20: 15797-15804. [Crossref]

23. Pitman MB, Centeno BA, Ali SZ, Genevay M, Stelow E, et al. (2014) Standardized terminology and nomenclature for pancreatobiliary cytology: The Papanicolaou Society of Cytopathology Guidelines. CytoJournal 11(Suppl 1): 3. [Crossref]

24. Nishimura A, Den N, Sato H, Takeda B (1973) Exfoliative cytology of the biliary tract with the use of saline irrigation under choledochoscopic control. Ann Surg 178: 594 599. [Crossref]

25. Othman MO, Guerrero R, Elhanafi S, Davis B, Hernandez J, et al. (2016) A prospective study of the risk of bacteremia in directed cholangioscopic examination of the common bile duct. Gastrointest Endosc 83: 151-157. [Crossref]

26. Sugimoto S, Matsubayashi H, Kimura H, Sasaki K, Nagata K, et al. (2015) Diagnosi of bile duct cancer by bile cytology: usefulness of post-brushing biliary lavage fluid. Endosc Int Open 3: E323-E328. [Crossref]

27. Tanaka R, Itoi T, Honjo M, Tsuchiya T, Kurihara T, et al. (2016) New digita cholangiopancreatoscopy for diagnosis and therapy of pancreaticobiliary diseases (with videos). J Hepatobiliary Pancreat Sci 23: 220-226. [Crossref]

28. Navaneethan U, Hasan MK, Kommaraju K, Zhu X, Hebert-Magee S, et al. (2016) Digital, single-operator cholangiopancreatoscopy in the diagnosis and management of pancreatobiliary disorders: a multicenter clinical experience (with video). Gastrointest Endosc 84: 649-655. [Crossref]

Copyright: (C2017 Motomura Y. This is an open-access article distributed under the terms of the Creative Commons Attribution License, which permits unrestricted use, distribution, and reproduction in any medium, provided the original author and source are credited. 